\title{
Effet In Vivo De Newbouldia laevis (Bignoniaceae) Sur Des Strongles Gastro-Intestinaux Des Moutons
}

\author{
Olounladé Abiodoun Pascal (PhD, Maître-Assistant) \\ Laboratoire Pluridisciplinaire de Kétou, Ecole de Gestion et d'Exploitation \\ des Systèmes d'élevages (EGESE), Université Nationale d'Agriculture, \\ 01 BP: 55 Porto-Novo, Bénin \\ Attakpa Yatchégnon Eloi, (PhD, Maître de Conférences) \\ Azando Erick Virgile Bertrand, (PhD, Maître-Assistant) \\ Faculté d'Agronomie, Université de Parakou, BP 123, Parakou, Bénin \\ Hounzangbé - Adoté Mawulé Sylvie, (PhD, Professeur

\section{Titulaire)}

Laboratoire d'Ethnopharmacologie et de Santé Animale (LESA), Faculté des

Sciences Agronomiques (FSA), Université d'Abomey-Calavi (UAC),

01 BP 526 Cotonou

\section{Hoste Hervé, (Directeur de recherche)}

Institut National de Recherche Agricole, / Ecole Vétérinaire de Toulouse, Université Paul Sabatier de Toulouse III, (France)

doi: 10.19044/esj.2017.v13n12p335 URL:http://dx.doi.org/10.19044/esj.2017.v13n12p335

\begin{abstract}
The in vivo evaluation of the effectiveness of pests leaves Newbouldia laevis (Bignoniaceae) was tested on gastrointestinal strongyles of Djallonké infested lambs naturally or artificially with larvae of Haemonchus contortus and Trichostrongylus colubriformis. In a controlled environment, the trial involved 15 lambs artificially infected and naturally infected some 14 was treated with the powder of $N$. laevis $(1.6 \mathrm{~g} / \mathrm{kg} \mathrm{BW})$. The parasitic balance sheet revealed that $N$. laevis limited larval Trichostrongylus installation colubriformis $(\mathrm{P}=0.01)$, whereas it had no effect on the larvae of $H$. contortus after artificial infestation. Following natural infection, this plant has limited $(\mathrm{P}=0.08)$ to the population of $H$. contortus adults was reduced by $55 \%$ in abomasum the experimental animals; this reduction is only $19 \%$ on the viability of adult worms of $T$. colubriformis ( $\mathrm{P}>0.05)$. However, $N$. laevis did not affect prolificacy to $H$. contortus females; but it has reduced by $11 \%$ T. colubriformis. Uncontrolled environment in a first trial, 16 animals were treated with 2 doses (3.2 or $4.8 \mathrm{~g}$ / $\mathrm{kg} \mathrm{BW}$ ) of dry powdered leaves. In a second test, the fresh leaves of $N$.
\end{abstract}


laevis were proposed ad libitum consumption three times a week for 45 days. The results show that leaves $N$. laevis significantly reduced $(\mathrm{P}<0.05)$ excretion of gastrointestinal strongyles eggs (80-90\%) in Djallonké lambs naturally infected regardless of the dose.

Keywords: Newbouldia laevis, in vivo, Haemonchus contortus, Trichostrongylus colubriformis, Benin

\section{Résumé}

L'évaluation in vivo de l'efficacité antiparasitaire des feuilles de Newbouldia laevis (Bignoniacées) a été testée sur les strongles gastrointestinaux des agneaux Djallonké infestés naturellement ou artificiellement par des larves de Haemonchus contortus et Trichostrongylus colubriformis. En milieu contrôlé, l'essai a porté sur 15 agneaux infestés artificiellement et 14 infestés naturellement dont certains ont été traités avec la poudre de $N$. laevis $(1,6 \mathrm{~g} / \mathrm{kg} \mathrm{PV})$. Le bilan parasitaire a révélé que $N$. laevis a limité l'installation des larves de $T$. colubriformis $(\mathrm{P}=0,01)$, alors qu'elle n'a eu aucun effet sur celle des larves de H.contortus après une infestation artificielle. Suite à l'infestation naturelle, cette plante a limité $(\mathrm{P}=0,08)$ la population de vers adultes de $H$. contortus qui a été réduite de $55 \%$ dans l'abomasum des animaux expérimentaux ; cette réduction n'est que de 19\% sur la viabilité des vers adultes de $T$. colubriformis $(\mathrm{P}>0,05)$. Toutefois, $N$. laevis n'a pas influencé la prolificité des vers femelles d'H.contortus ; mais elle l'a réduite de $11 \%$ chez T. colubriformis. En milieu non contrôlé dans un premier essai, 16 animaux ont été traités avec 2 doses (3,2 ou 4,8g/kg PV) de feuilles sèches réduites en poudre. Dans un deuxième test, les feuilles fraîches de $N$. laevis ont été proposées en consommation ad libitum trois fois par semaine durant 45 jours. Les résultats montrent que les feuilles de $N$. laevis ont réduit significativement $(\mathrm{P}<0,05)$ l'excrétion des œufs de strongles gastro-intestinaux $(80-90 \%)$ chez les agneaux Djallonké infestés naturellement quelle que soit la dose.

Mots-clés : Newbouldia laevis, in vivo, Haemonchus contortus, Trichostrongylus colubriformis, Bénin

\section{Introduction}

L'utilisation de plantes médicinales à des fins thérapeutiques, pratique multiséculaire, connaît depuis ces deux dernières décennies une certaine réémergence. En effet, le regain d'intérêt pour les plantes après plus d'un demi siècle d'usage massif des molécules chimiques de synthèse, s'explique non seulement par la paupérisation croissante des populations des pays du Sud, mais aussi et surtout par le fait que les molécules de synthèse ont tôt fait 
de montrer leurs limites : les résidus non dégradables, les cas de résistance aux anthelminthiques classiques sont fréquents ce qui diminue considérablement leur efficacité et pose des problèmes de maîtrise du parasitisme (Jackson et Coop, 2000 ; Kaplan, 2004; Azando, 2011). Ces éléments ont conduit à s'interroger sur la durabilité des systèmes de gestion, fondés sur l'emploi presque exclusif de la chimiothérapie et à envisager de nouvelles méthodes de lutte antiparasitaire.

L'emploi de nouvelles substances vermifuges, comme moyen de lutte contre les parasites gastro-intestinaux, est une approche prometteuse qui repose sur l'exploitation des plantes ou des substances naturelles dotées de propriétés anthelminthiques. Il s'agit, dans les pays tempérés, de plantes riches en tanins (Molan et al., 2000 ; Athanasiadou et al., 2001 ; Niezen et al., 1998a - b ; Paolini et al., 2002 ; 2003a - b) et dans les zones tropicales de plantes bio actives utilisées en pharmacopée traditionnelle (Kasonia \& Ansey, 1994 ; Mbarubukeye, 1994 ; Abadome \& Geerts, 1993 ; Bognounou, 1993 ; Hammond et al., 1997 ; Tamboura et al., 1998 ; N'Fi et al., 1999). Cette alternative offre la perspective de moyens de lutte peu coûteux, et surtout accessibles aux éleveurs et agro-éleveurs car issue des ressources locales. Les Newbouldia de la famille des Bignoniacées sont répartis partout en Afrique. L'espèce Newbouldia leavis (Berhaut, 1974 ; de Souza, 1988; Olounladé, 2011), objet de cette étude, pousse (abondamment) dans les forêts de l'Afrique de l'Ouest, du Sénégal au Zaïre (Pauwel et Nzayili, 1993). On rencontre cette plante dans les anciens lieux de fétiches où il constitue une plante de haie de clôture. Ses feuilles interviennent souvent dans les cérémonies traditionnelles en Afrique pour la purification. En pharmacopée humaine, l'écorce et les racines de la plante entrent dans plusieurs recettes pour soigner les affections respiratoires, gastriques (Adjanohoun et al, 1989) ou les maladies cardiovasculaires (Aklikokou et Gbeassor, 1997) ou encore pour ses propriétés antimicrobiennes ou antibactériennes (Eyong et al., 2006). En pharmacopée vétérinaire, il est cité par les éleveurs comme ayant des propriétés antiparasitaires (Hounzangbé Adoté, 2000; Alowanou, 2016).

In vitro, des extraits alcooliques $(60 \%)$ de feuilles sèches Newbouldia laevis ont limité l'éclosion des œufs, la migration larvaire et la viabilité des vers adultes de Haemonchus contortus (Hounzangbé - Adoté et al., 2005a) et de Trichostrongylus colubriformis (Hounzangbé - Adoté et al., 2005b) à des doses variant de 150 à $1200 \mu \mathrm{g} / \mathrm{mL}$.

Le but de cette étude est d'évaluer in vivo, l'efficacité anthelminthique de $N$. laevis sur les strongles gastro-intestinaux des ovins afin de mieux valoriser cette plante locale citée comme antiparasitaire par les éleveurs. 


\section{Matériel et méthodes}

Les feuilles de $N$. leavis (Bignoniaceae) ont été récoltées de Juillet à Octobre 2007 (petite saison des pluies) et authentifiées à 1'Herbier National de la Faculté des Sciences et Techniques de 1'Université d'Abomey-Calavi. Elles ont été séchées en salle climatisée $\left(20^{\circ} \mathrm{C}\right)$ pendant 5 jours et sont ensuite réduites en poudre dans un broyeur.

\section{Test en milieu contrôlé}

A la ferme de la Faculté des Sciences Agronomique de 1'Université d'Abomey-Calavi, deux (2) essais ont été menés pour évaluer l'efficacité des feuilles de $N$. laevis sur l'installation des larves infestantes $\mathrm{L}_{3}$ et sur la viabilité des vers adultes des deux espèces de nématdes, parasites gastrointestinaux (H. contortus et $T$. colubriformis).

\section{Essai sur l'installation des larves}

Quinze agneaux Djallonké, âgés de 2 à 6 mois avec un poids moyen de $7,5 \pm 1,5 \mathrm{~kg}$ ont été infestés artificiellement par 3000 larves des deux espèces. Sept agneaux ont été traités $(1,6 \mathrm{~g} / \mathrm{kg}$ de Poids vif) pendant 3 jours $\left(\mathrm{J}_{-1}\right.$ à $\left.\mathrm{J}_{+1}\right)$ au moment de l'infestation afin d'évaluer l'effet de la poudre de $N$. laevis sur l'installation des larves de strongles digestifs. Deux semaines après le traitement, tous les animaux ont été abattus et les vers adultes $d^{\prime} H$. contortus ont été dénombrés dans $10 \%$ du volume de l'abomasum et ceux de T. colubriformis dans l'intestin grêle.

\section{Essai sur la population des vers adultes}

Quatorze agneaux infestés naturellement ont été mis en claustration pendant 31 jours et répartis en deux lots de 7 animaux (témoin et traités). Du $14^{\mathrm{e}}$ au $16^{\mathrm{e}}$ jours, les animaux du lot expérimental ont été traités à la poudre de feuilles de $N$. laevis à la dose de $1,6 \mathrm{~g} / \mathrm{kg}$ de Poids vif. Les prélèvements coprologiques bihebdomadaires ont permis d'évaluer l'impact de la plante sur l'excrétion des œufs. Tous les animaux ont été sacrifiés 15 jours après le traitement pour un bilan parasitaire et les vers adultes ont été dénombrés dans un aliquot de $10 \%$ du contenu de l'estomac $(H$. contortus) ou de l'intestin grêle (T. colubriformis). Dix vers femelles par espèce ont été récupérés chez tous les animaux puis conservés dans de l'alcool à $70^{\circ}$. Chaque femelle est ensuite écrasée dans une solution d'hypochlorite de sodium (2\%) dilué, afin de compter les œufs in utero, selon la technique de Kloosterman et al. (1978).

Du sang prélevé une fois par semaine, au niveau de la veine auriculaire dans des microtubes à héparine, centrifugé à $1200 \mathrm{tr} / \mathrm{min}$ pendant $5 \mathrm{~min}$ a permis de déterminer l'hématocrite des animaux. 


\section{Test en milieu non contrôlé}

Deux tests ont été menés dans les conditions d'élevage en milieu paysan dans un village situé à $15 \mathrm{~km}$ d'Abomey-Calavi, sur des animaux de même race que ceux utilisés en milieu contrôlé.

Dans un premier essai, 28 animaux ont été mis aux piquets au cours de la petite saison des pluies. Douze n'ont reçu aucun traitement (Lot témoin) et 16 ont été répartis en 2 lots de 8 animaux chacun. Le premier lot a été traité avec $3,2 \mathrm{~g} / \mathrm{kg}$ PV de feuilles séchées et moulues et le deuxième lot a eu un traitement avec $4,8 \mathrm{~g} / \mathrm{kg}$ PV de $\mathrm{J}_{14}$ à $\mathrm{J}_{16}$ après leur mise en stabulation et suivis encore pendant 15 jours. Les prélèvements coprologiques hebdomadaires ont permis de déterminer l'effet de la plante sur l'excrétion des œufs.

Dans le deuxième essai, l'objectif était de déterminer la quantité ingérée par les animaux en conditions d'élevage afin d'estimer la dose adéquate. Dix (10) moutons témoins et six (6) expérimentaux ont été mis au piquet au cours de la même période. Les animaux expérimentaux ont reçu trois (3) fois par semaine des feuilles de $N$. laevis comme fourrage à satiété. La quantité de feuilles ingérées a été déterminée par la prise de poids du fourrage servi et des refus chaque jour pendant 5 jours. Le poids des animaux a été pris au début et à la fin de l'expérimentation. Le gain moyen quotidien (GMQ) exprimé en gramme est le rapport de la différence de poids initial et de poids final par la durée de l'expérience.

Les prélèvements coprologiques bimensuels ont permis par la méthode de Mc Master (Raynaud, 1970) d'évaluer l'impact de la plante sur l'excrétion des œufs pendant 45 jours.

\section{Résultats et discussion Etude en station \\ Effet sur l'installation des larves $\mathrm{L}_{3}$}

Le taux d'installation des larves, est relativement faible quelque soit l'espèce. Ce taux semble plus élevé pour $H$. contortus (30\%) que pour $T$. colubriformis $(13 \%)$ et varie beaucoup d'un animal à l'autre. L'autopsie des animaux infestés artificiellement a révélé que $N$. laevis n'aurait pas d'effet in vivo sur l'installation des larves d' $H$. contortus, alors que de nombre des $T$. colubriformis installé a diminué de $71 \%(\mathrm{p}<0,05)$ après le traitement par la plante dans les mêmes conditions (Tableau 1).

Tableau 1 : Effet sur l'installation des larves infestantes $\left(\mathrm{L}_{3}\right)$ (Comptage des vers après abattage)

\begin{tabular}{cccc}
\hline Lots & H. contortus & T. colubriformis & Total des vers \\
\hline Témoin & $347,2 \pm 240,3$ & $302,5 \pm 255,6$ & $\mathbf{6 4 9 , 7} \pm \mathbf{3 6 3 , 7}$ \\
\hline Traité & $644,3 \pm 500,0$ & $87,1 \pm 105,6$ & $\mathbf{7 3 1 , 4} \pm \mathbf{5 4 7 , 9}$ \\
\hline
\end{tabular}




\section{Effet sur la population de vers adultes Excrétion des œufs}

Après le traitement des animaux par la poudre de feuilles de $N$. laevis, le niveau d'excrétion des œufs de strongles a significativement diminué dans le lot expérimental (Figure 1). Cette réduction sensible dès 48 heures après le début du traitement $\left(\mathrm{J}_{17}\right)$ a atteint le maximum $94 \%$ à $\mathrm{J}_{23}$ avec une différence très significative $(\mathrm{P}<0,001)$ entre les animaux traités et les témoins de $\mathrm{J}_{17}$ à $\mathrm{J}_{31}$.

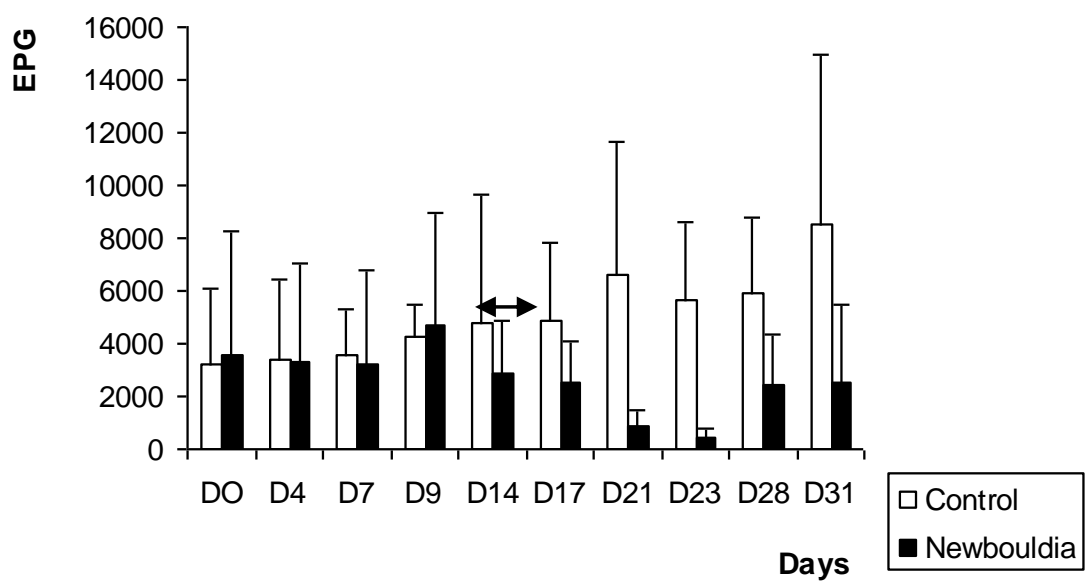

Figure 1 : Variation de l'excrétion des œufs de strongles chez des animaux traités (D14D16) par la poudre de Newbouldia laevis en station

\section{Bilan parasitaire}

Une tendance à la limitation de la charge parasitaire chez les animaux naturellement infestés traités a été enregistrée $(\mathrm{P}<0,08)$ : Le nombre de vers adultes d' $H$. contortus a diminué de $55 \%$ (Tableau 2). Par contre pour $T$. colubriformis, la réduction enregistrée n'est que de $19 \%$. Le sex ratio est de l'odre de $60 \%$ de femelles et $40 \%$ de mâles quel que soit l'espèce et le traitement.

$\underline{\text { Tableau } 2}$ : Effet in vivo sur la viabilité des vers adultes (Comptage des vers adultes après abattages des animaux)

\begin{tabular}{llccc}
\hline Lots & Vers & H. contortus & T. colubriformis & Total \\
\hline \multirow{2}{*}{ Témoin } & Vers mâles & $232,9 \pm 150,3$ & $97,1 \pm 52,82$ & $330 \pm 139$ \\
\cline { 2 - 5 } & Vers femelles & $417,1 \pm 212,4$ & $127,14 \pm 53,14$ & $544,3 \pm 192$ \\
\cline { 2 - 5 } & Total de vers adultes & $650,0 \pm 336,9$ & $224,28 \pm 95,02$ & $\mathbf{8 7 4 , 3} \pm \mathbf{3 1 0}$ \\
\hline \multirow{2}{*}{ Traité } & Vers mâles & $91,7 \pm 62,1$ & $80 \pm 56,57$ & $171,7 \pm 79,6$ \\
\cline { 2 - 5 } & Vers femelles & $203,3 \pm 71,7$ & $101,67 \pm 83,0$ & $305 \pm 93$ \\
\hline
\end{tabular}




\section{La prolificité des vers femelles}

Le dénombrement des œufs in utéro des vers femelles de $H$. contortus montre que ces vers présentent la même prolificité aussi bien chez les animaux traités que chez les témoins. N.laevis n'a donc pas d'effet sur la prolificité des vers. Chez les femelles de $T$. colubriformis la prolificité est réduite de $11 \%$ (tableau 3 ).

Tableau 3 : Variation de la prolificité des vers adultes de $H$. contortus

\begin{tabular}{ccc}
\hline Lots & H. contortus & T. colubriformis \\
\hline Témoin & $747,33 \pm 81,65$ & $105,25 \pm 8,72$ \\
\hline Expérimental & $711,72 \pm 85,79$ & $93,63 \pm 14,64$ \\
\hline
\end{tabular}

\section{Hématocrite}

La figure 2 montre que le volume de globules rouges a varié au cours de l'expérience. Avant le traitement, le niveau d'hématocrite est semblable dans les deux lots. Après le traitement, il a été observé de $J_{18}$ à $J_{31}$ une tendance à la diminution de l'hématocrite dans le lot témoin $(\mathrm{P}=0,08)$.

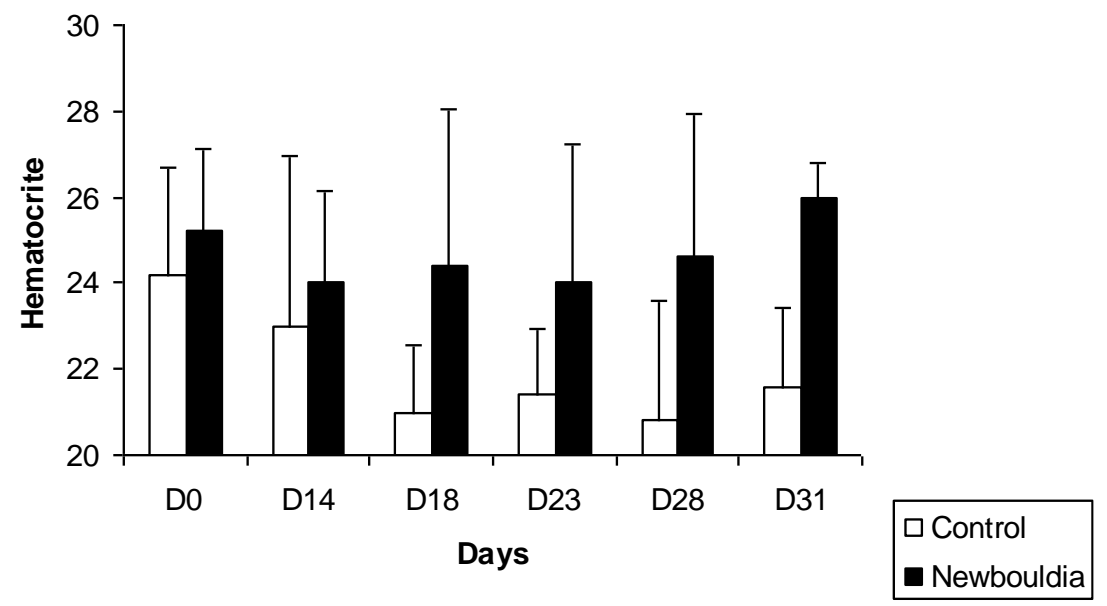

Figure 2: Variation de l'hématocrite des animaux traités ou non avec la poudre de Newbouldia en station

\section{Etude en milieu non contrôlé}

\section{Efficacité de la poudre de feuilles de $\mathbf{N}$. laevis}

L'utilisation des doses plus fortes de poudre de $N$. laevis en condition d'élevage a confirmé la baisse (Figure 3) observée précédemment, avec $85 \%$ $(3,2 \mathrm{~g} / \mathrm{kg} \mathrm{PV})$ et $88 \%(4,8 \mathrm{~g} / \mathrm{kg} \mathrm{PV})$ et une différence significative $(\mathrm{P}<0,05)$ entre les animaux traités et les non traités à partir d'une semaine après le 
traitement $\left(\mathbf{J}_{21}\right)$. Toutefois, cette réduction n'est pas été influencée par la dose du traitement.

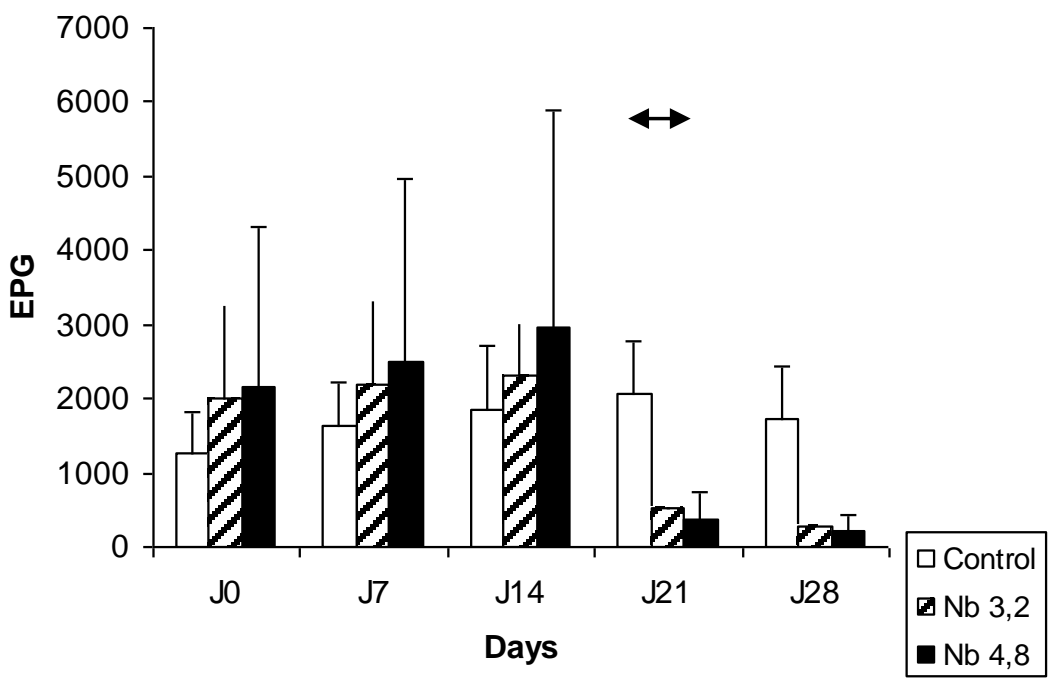

Figure 3 : Variation de l'excrétion des œufs de strongles chez des animaux traités à la poudre de Newbouldia laevis en milieu paysan

\section{Efficacité des feuilles fraîches de N. laevis}

En milieu paysan, l'ingestion des feuilles de $N$. laevis mesurée est de $1,28 \pm 0,5 \mathrm{~kg}$. Cette ingestion a régresséavec le temps et est passée de 1,5 à $0,8 \mathrm{~kg} /$ jour et par animal. Toutefois, il a été noté une prise de poids des animaux expérimentaux comparables aux témoins (Tableau 4). Cette consommation a induit dès les premières semaines $\left(\mathrm{J}_{13}\right)$ une réduction de l'excrétion moyenne des oeufs à près de $67,5 \%$ (Figure 4 ). Cette baisse a atteint $89 \%$ après 6 semaines de distribution répétée avec une différence significative $(\mathrm{P}<0,01)$ entre les animaux traités et les témoins.

Tableau 4 : Variations pondérales (Infestation naturelle)

\begin{tabular}{llll}
\hline Lots & Poids initial $(\mathbf{K g})$ & Poids final $(\mathbf{K g})$ & GMQ $(\mathbf{g})$ \\
\hline Témoin & $9,96 \pm 3,15$ & $10,78 \pm 3,19$ & $18,15 \pm 8,93$ \\
\hline N. laevis & $10,0 \pm 1,45$ & $11,84 \pm 1,05$ & $40,89 \pm 32,03$ \\
\hline
\end{tabular}




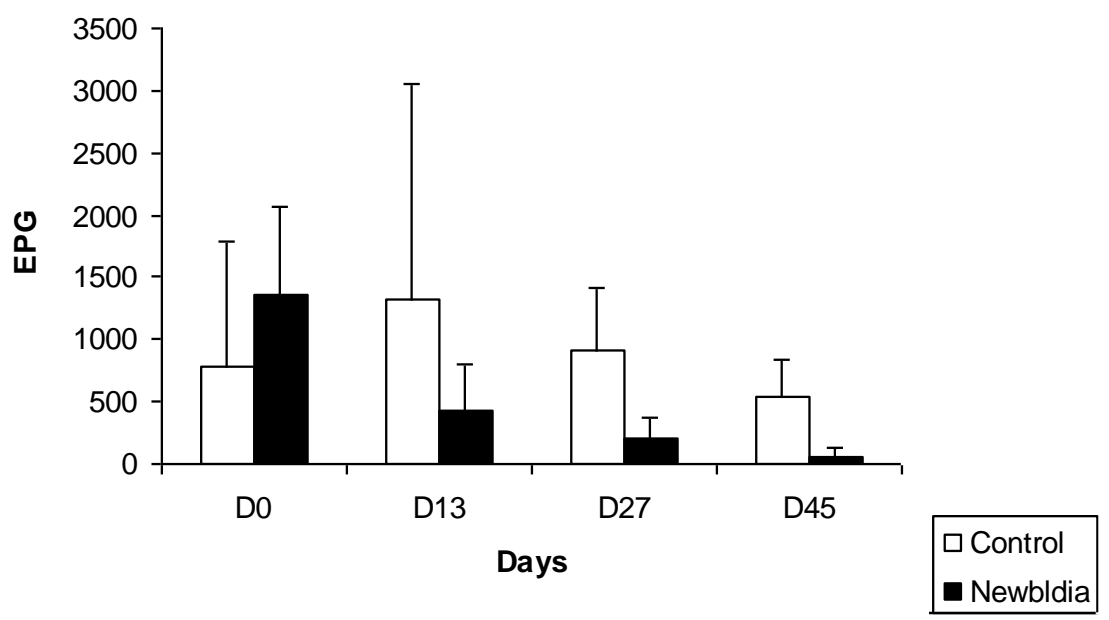

Figure 4 : Variation de l'excrétion des œufs de strongles traités par la distribution répétée de la feuille fraîche de $N$. laevis en milieu paysan

In vitro, des extraits alcooliques (60\%) de feuilles sèches de Newbouldia laevis ont limité l'éclosion des œufs, la migration larvaire et la viabilité des vers adultes de H.contortus (Hounzangbé - Adoté et al., 2005a) et de T. colubriformis (Hounzangbé - Adoté et al., 2005b) à des doses variant de 150 à $1200 \mu \mathrm{g} / \mathrm{mL}$. Les essais in vitro permettent de tester en peu de temps, les propriétés anthelminthiques de différentes plantes sur les différents stades parasitaires et d'analyser les mécanismes d'action de ces plantes. Ces tests mesurent directement les effets anthelminthiques sur les processus comme ceux de l'éclosion des œufs, le développement et la motilité des parasites (Varady et Corba, 1999). Toutefois, ces essais sont mal adaptés pour tester les mécanismes indirects qui tiennent compte de la réponse de l'hôte. Il se pose donc de multiple questions sur la validité des facteurs maîtrisés in vitro tels que les concentrations appliquées, les techniques d'obtention des extraits. Dans les conditions physiologiques, plusieurs facteurs peuvent modifier la réponse de l'organisme animal. L'animal n'étant pas qu'un réceptacle amorphe de parasite, il peut interagir avec la substance donnée par des phénomènes physiologiques telles que le métabolisme et la réponse immunitaire. Ainsi, le développement des larves infestantes en adultes, la fécondité de ces vers adultes ou l'excrétion des œufs varient significativement d'un animal à l'autre et chez un même animal d'un prélèvement à l'autre (Gasbarre, 1997). D'où la nécessité de passer aux tests in vivo.

In vivo, $N$. laevis a limité l'installation des larves de T.colubriformis. Cette limitation serait certainement due à l'effet de la plante à immobiliser ces larves. En effet, nos travaux antérieurs ont montré in vitro que $N$. laevis 
inhibe de l'ordre de $80 \%$ la migration larvaire de cette espèce (HounzangbeAdoté et al., 2005 b). Par contre sur $H$. contortus, cette inhibition bien qu'effective a été moins forte (50\%) (Hounzangbe-Adoté et al., 2005 a). D'autres travaux en cours révèlent que cette plante empêche significativement in vitro le dégainement des larves de T. colubriformis. D’autres auteurs (Bahuaud et al., 2006 ; Brunet \& Hoste, 2006) ont montré que certaines plantes riches en tanin avaient la propriété de bloquer le dégainement des larves sans lequel le cycle de vie du parasite ne pourra se poursuivre.

La diminution de l'excrétion des œufs observée in vivo peut être due soit à la mortalité des vers adultes, soit à une baisse de prolificité des vers femelles. Selon les résultats cet effet est plus sur la viabilité des vers adultes de $H$. contortus que sur leur prolificité. L'action de $N$. laevis semble différente d'autres plantes tropicales telle que Zanthoxylum zanthoxylö̈des communément appelé fagara, qui a plus un effet sur la prolificité des vers adultes de $H$. contortus que sur leur viabilité (Hounzangbé-Adoté et al., 2005c). L'efficacité de la poudre des feuilles de N. laevis, in vivo confirme les études in vitro sur la viabilité des vers adultes de $H$. contortus, (Hounzangbé-Adoté et al., 2005a, b). Alors que cette concordance n'a pas été notée pour son action sur $T$. colubriformis. Il faut noter que les effets observés peuvent être différents de ceux in vitro notés qui indiquent des effets directs. Nous pouvons aussi émettre l'hypothèse qu'une dégradation des substances actives, notamment dans le rumen exige l'utilisation in vivo des doses plus fortes que in vitro. Car, si la dose de $1,6 \mathrm{~g} / \mathrm{kg}$ sur trois (3) jours utilisé dans cet essai, est équivalente aux doses utilisées in vitro $(200 \mu \mathrm{g} / \mathrm{mL})$ en tenant compte du rendement à l'extraction alcoolique $(12,8 \%)$ de $N$. laevis, $(600 \mu \mathrm{g} / \mathrm{mL}=0,6 \mathrm{~g} / \mathrm{kg} ; 12,8 \%$ de $1,6 \mathrm{~g}=0,2 \mathrm{~g}$ x 3 jours $=0,6 \mathrm{~g})$, elle semble relativement faible d'où la nécessité de tester des doses plus fortes dans le deuxième essai. Cependant, l'utilisation du double et du triple de cette dose en milieu paysan, tout restant efficace par la réduction significative de l'excrétion des œufs n'a révélé aucun effet dose. Malheureusement l'abattage d'un nombre d'animaux statistiquement représentatif n'a pas été possible en milieu paysan ; les éleveurs ne pouvaient pas déstocker leur troupeau.

La mesure de la quantité de $N$. laevis ingérée par animal a permis de déterminer en condition réelle la dose naturelle. Ains, en tenant compte de la teneur en matière sèche de cette plante $(40 \%)$ et du rendement à l'extraction $(12,8 \%)$, la quantité de plante ingérée par les animaux consommée serait équivalente à la dose de $6,65 \mathrm{~g} / \mathrm{kg}$ d'extrait alcoolique soit 1,5 à 2 fois la dose administrée $(40 \% \mathrm{MS}$ de $1,3 \mathrm{~kg}=520 \mathrm{~g}$ pour $10 \mathrm{~kg}$ poids des animaux ; soit $52 \mathrm{~g} / \mathrm{kg}, 12,8 \%$ extrait de $52 \mathrm{~g}=6,65 \mathrm{~g}$ ). Dans la mesure où aucun effet toxique n'été observé, ni signalé dans la littérature, cette dose ne pourrait 
qu'améliorer l'effet de la plante ; Ce qui s'est traduit par une réduction plus significative de l'excrétion des œufs de strongles et un gain de poids conséquent sans une amélioration des conditions d'élevage. Car selon la table d'évaluation alimentaire en zone tropicale (Osuji et al., 1993), les animaux en conditions d'élevage, ingèrent en moyenne $50 \mathrm{~g} \mathrm{MS} / \mathrm{kg}$ de poids vif corporel de fourrage par jour. En se référant aux travaux en station, il a été observé aussi que la poudre de feuille de $N$. laevis permet de maintenir le volume de globules rouges dans le sang.

Les auteurs expliquent les propriétés antifongiques et antimicrobien de $N$. laevis par sa richesse en quinone et en alcaloïdes associée à des pigments : Newbouldiquinone (Eyong et al., 2006) ou ceramide (Eyong et al. 2005). Un total de 15 substances ont été isolé des racines de $N$. laevis. La majorité de ces composés sont des dérivés de la déhydroiso-a-lapachone, une naphtoquinone déjà connue chez d'autres espèces de la famille des Bignoniacées. De petites quantités de 3 naphthaquinones ont été isolées de l'écorce des racines de $N$. laevis: lapachol deshydro- $\alpha$ - lapachone et 3 hydroxypyrol $(1,2 b)$, pyrazol alkaloid, newbouldine (Houghton et al., 1994). Un nouveau type structural d'alcaloides extrait de $N$. laevis récolté au Nigeria près de Anambra state a été nommé newbouldine $\left(\mathrm{C}_{12} \mathrm{H}_{14} \mathrm{~N}_{2}\right)$ par Gafner et al. (1998). Quatre composés supplémentaires ont été décelés dans l'extrait dichlorométhanique de la racine, la withasomnine, la newbouldine et leurs dérivés 4'-hydroxylés (Gafner et al., 1996). L'extrait au butanol de la racine a permis d'isoler trois glycosides phénylpropanoiques, dont le newbouldioside qui s'est avéré être un nouveau produit naturel (Gafner et al., 1997). Toutefois, les molécules responsables de l'effet anthelminthique sont encore mal connues. Il ressort des résultats de l'analyse phytochimique (Olounladé, 2005) que $N$. laevis renferme les familles des composés comme : Les tanins, les flavonoïdes, les anthocyanes, les dérivés quinoniques, les saponosides, les mucilages, les stéréoïdes et triterpenoïdes. La présence des tanins dans la poudre de feuilles de $N$. laevis pourrait justifier les propriétés anthelminthiques observées. Les tanins condensés ayant des effets bénéfiques sur la digestion, en particulier chez les ruminants auraient des propriétés antiparasitaires (Lu et al., 2000). De travaux réalisés avec des légumineuses fourragères (Molan et al., 2000) ainsi qu'avec des extraits de plantes ligneuses (Paolini et al., 2002) confirment le rôle joué par les tanins condensés (Molan et al., 2003; Paolini et al., 2002 ) dans la lutte antiparasitaire. Molan et al. (2002) ont évalué la quantité de tanin condensé dans la ration de maintenance des moutons de fourrages à environ 30 à $120 \mathrm{~g}$ à travers le tractus digestif par jour, Al Qarawi et al. (2001), à des doses plus faibles chez les moutons traités avec le latex de Calotropis procera à 0,01 ou $0,02 \mathrm{~mL} / \mathrm{kg}$ de poids vif, ont enregistré une excrétion des œufs significativement réduite, mais pas supprimée complètement. Ceci est en 
adéquation avec nos résultats dans lesquels la plante testée n'a éliminé que partiellement l'infestation parasitaire.

\section{Conclusion}

In vivo, Les feuilles de $N$. laevis ont réduit significativement l'excrétion des œufs de strongles chez des moutons infestés naturellement contrôlé ou non. La diminution d'excrétion fécale des œufs de parasites après ingestion des plantes testées indique la portée épidémiologique de ces résultats par leurs conséquences possibles sur la contamination du pâturage. Globalement, les effets de la plante paraissent semblables avec les deux types de tests: Les données in vivo tendent à confirmer ces résultats in vitro. L'utilisation des feuilles de $N$. laevis comme anthèlminthique en milieu paysan est justifiée.

\section{Remerciements}

Les auteurs remercient les vendeuses de plantes médicinales, des éléveurs et de tous les acteurs ayants participés aux différents travaux de recherche. Les remerciement vont également à l'endroit de l'Institut National de la Recherche Agricole du Bénin pour son appui financier.

\section{References:}

1. Abadome, F., and Geerts, S. (1993). Ambrosia maritima. Connaissances actuelles et perspectives. In : Kasonia, K., and Ansey, M. (1994). Métissage en santé animale de Madagascar à Haïti. Edition Botanique Presse Universitaire de Namur, CTA, ACCT, p 296 - 303.

2. Adjanohoun, E.J., Adjakidjè, V., Ahyi, M.R.A., Ake, A.L., Akoegninou, A., d'Almeida, J., Akpovo, F., Bouke, K., Chadare, M., Cusset, G., Dramane, K., Eyme, J., Gassita, J.N., Gbaguidi,N., Goudoté, E., Guinko, S., Hougnon, P., Issa, L.O., Keita, A., Kiniffo, H.V., Kone, B.D., Musampa, N.A., Saadou, M., Sodogandji, T.H., de Souza, S., Tchabi, A., Zinsou, D.C., and Zohoun, T.H. (1989). Contribution aux études ethnobotaniques et floristiques en République Populaire du Bénin. Médecine traditionnelle et pharmacopée. ACCT, p. 895.

3. Aklikokou, A.K., and Gbeassor, M. (1997). Contribution à l'étude des propriétés pharmacologiques des extraits totaux de quelques plantes antihypertensives Ann. Univ. Bénin, Sér. Sciences, Tome XIII, 1997, pp. $26-39$.

4. Al Qarawi, A.A., Mahmoud, O.M., Sobaih, M.A., Haroun, E.M., and Adam, S.E.I. (2001). A preliminary study on anthelmintic activity of 
Calotropis procera latex against Haemonchus contortus infection in Najdi sheep. Vet. Res. Comm., 25: 61-70.

5. Alowanou, G.G. (2016). Utilisation de Bridelia ferruginea Benth, Mitragyna inermis (Willd.) Kuntze et Combretum glutinosum Perr. ex DC., dans le contrôle de Haemonchus contortus chez trois types génétiques d'ovins au Bénin. Thèse de doctorat unique de l'Université d'Abomey-Calavi, 250p

6. Athanasiadou, S., Kyriazakis, I., Jackson, F., and Coop R.L. (2001). Direct anthelmintic effects of condensed tannins towards different gastrointestinal nematodes of sheep: in vitro and in vivo studies. Vet. Parasitol., 99: 205 - 219.

7. Azando, E.V.B. (2011). Facteurs de variabilité de l'activité anthelminthique de Newbouldia laevis et de Zanthoxylum zanthoxylö̈des sur les nématodes parasites gastro-intestinaux des petits ruminants. Thèse de doctorat de l'Université d'Abomey-Calavi, Bénin, 296p

8. Bahuaud, D., Martinez-Ortiz, C., De Montellano, C.S., Prevot, F., Torres-Acosta, F., Fouraste, I., and Hoste H. (2006). Effects of four tanniferous plants extracts on the in vitro exsheathment of third stage larvae of parasitic nematodes. Parasitology, 132: 1-10.

9. Berhaut, S. (1974). Flore illustrée du Sénégal. Tome 2, Diffusion Claireafrique, Dakar - Livre africain 13, rue de Sevre Paris, 695 p.

10. Bognonou, O. (1993). Réflexion sur les thérapeutiques en soins de santé animale et état des connaissances ethnobotaniques au Burkina Faso. In: Kasonia, K., and Ansey, M. (1994). Métissage en santé animale de Madagascar à Haïti. Edition Botanique Presse Universitaire de Namur, CTA, ACCT, 296 p

11. Brunet, S., and Hoste, H. (2006). Monomers of condensed tannins affect the larval exsheathment of parasitic nematodes of ruminants. Journal of Agricultural and Food Chemistry, 54 : 7481 - 7487. De Souza, S. (1988). Flore du Bénin : Noms des plantes dans les langues nationales béninoises. Imprimerie Notre dame, Cotonou, 424p.

12. Eyong, K.O., Folefoc, G.N., Kuete, V., Beng, V.P., Krohn, K., Hussain H., Nkengfack, A.E., Saeftel, M., Sarite, S.R., and Hoerauf, A. (2006) Newbouldiaquinone A: A naphthoquinone-anthraquinone ether coupled pigment, as a potential antimicrobial and antimalarial agent from Newbouldia laevis. Phytochemistry. 67(6):605-9.

13. Eyong, K.O., Krohn, K., Hussain, H., Folefoc, G.N., Nkengfack, A.E., Schulz, B., and $\mathrm{Hu}, \mathrm{Q}$. (2005). Newbouldiaquinone and newbouldiamide: a new naphthoquinone-anthraquinone coupled pigment and a new ceramide from Newbouldia laevis. Chem Pharm Bull (Tokyo). 53(6):616-9. 
14. Gafner, S., Wolfender, J.L., Nianga, M., and Hostettmann, K. (1997). Phenylpropanoid glycosides from Newbouldia laevis roots, 44(4):687-90

15. Gafner, S., Wolfender, J.L., Nianga, M., and Hostettmann K. (1998). A naphtoquinone from Newbouldia laevis roots. Phytochem, 48: 215 $-216$.

16. Gafner, S., Wolfender, J.L., Nianga, M., Stoeckli, E.H., and Hostehmann K. (1996). Antifungal and antibacterial naphtoquinones from Newbouldia laevis roots. Phytochem. 42: 1315 - 1320.

17. Gasbarre, L.C., (1997). Do fecal egg counts accurately reflect worm numbers in cattles? USDA Technical Bulletin.

18. Hammond, J.A., Feiling, D., and Bishop, S.C. (1997). Prospects for plant anthelminthics in tropical veterinary medicine. Vet. Res. Comm., 21: $213-228$

19. Hansen, J., and Perry, B. (1995). Epidémiologie, Diagnostic et Prophylaxie des Helminthoses des ruminants domestiques : $7^{\text {ème }}$ Edition.

20. Houghton, P.J., Pandey, R., and Hawkes, E.J. (1994). Naphthaquinones and an alkaloid from roots of Newbouldia laevis. Phytochem., 35:1602 - 1603.

21. Hounzangbé-Adoté, M.S. (2000). Pharmacopée en médecine vétérinaire au sud du Bénin (cas des ovins et caprins). Actes du Congrès International sur les Origines des pharmacopées traditionnelles et élaboration des pharmacopées savantes du 11 au 13 mai 2000 p $376-379$.

22. Hounzangbé-Adoté, M.S., Paolini, V., Fouraste, I., Moutaïrou, K., and Hoste, H. (2005). In vitro effects of four tropical plants on three stages of the parasitic nematodes, Haemonchus contortus. Research in Veterinary Science, $78: 155-160$.

23. Hounzangbé-Adoté, M.S., Zinsou, F.E., Affognon, K.J., Koutinhouin, B., Adamou-N'Diaye, M., and Moutaïrou, K. (2001). Efficacité antiparasitaire de la poudre des graines de papaye (Carica papaya) sur les strongles gastro-intestinaux des moutons Djallonké au sud du Bénin. Rev. Elev. Méd. Vét. Pays trop. 54 (3-4) 225 - 229.

24. Hounzangbé -Adoté, M.S., Zinsou, F.E., Hounkpè, V., Moutaïrou, K., and Hoste H. (2003). Propriétés antiparasitaires de quelques plantes locales sur des nématodes gastro-intestinaux des ovins Djallonké en milieu réel. Journées Scientifiques de l'INRAB, Abomey-Calavi du 11 au 13 décembre 2003.

25. Hounzangbé-Adoté, M.S., Moutairou, K., and Hoste H. (2005a). In vitro effects of four tropical plants on three stages of the parasitic 
nematodes, Trichostrongylus colubriformis. J. of Helminthology, 79 : 29-3

26. Hounzangbé-Adoté, M.S., Zinsou F.E., Hounkpè V., Moutairou, K., and Hoste, H. (2005b). In vivo effects of fagara leaves on sheep infected with gastro-intestinal nematodes. Tropical Animal Health and Production, 37: 205 - 214.

27. Jackson, F. and Coop R.L. (2000). The development of anthelmintic resistance in sheep nematodes. Parasitol., 120: 95 - 107.

28. Kaplan, R.M., (2004). Drug resistance in nematodes of veterinary importance: a status report. Trends Parasitol. 20: $477-481$.

29. Kasonia, K., and Ansey, M., (1994). Métissage en santé animale de Madagascar à Haïti. Actes du séminaire d'Ethnopharmacopée vétérinaire, Ouagadougou, Burkina Faso, 1993 - Edition Botanique Presse Universitaire de Namur - Belgique, CTA, ACCT, p. 21 - 28.

30. Kloosterman, A., Albers, G.A.A., and Van Den Brink, R. (1978). Genetic variations among calves in resistance to nematode parasites. Veterinary Parasitology, 4, 353-368

31. Lu, Y., Sun, Y., Yeap F., Mc Nabb, W.C., Molan, A.L. (2000). Polyphenols condensed tannins and other natural products in onobrychis Vicifolia (Sain foin). J. Agric Food Chem., 48: 3440 3447

32. Mbarubukeye, S. (1994). La recherche sur la médecine Vétérinaire traditionnelle au Rwanda. In : Kasonia K. \& Ansey M. (eds) Métissage en santé animale de Madagascar à Haïti, Actes du Séminaire d'ethnopharmacologie vétérinaire, Ouagadougou, Burkina Faso, 1994. Edition Botanique, Presse Universitaire de Namur, CTA, ACCT, 253 - 266.

33. Molan, A.L., Alexander, R.A., Brookes, I.M., and Mc Nabb, W.C. (2000). Effects of an extract from sulla (Hedysarum coronarium) containing condensed tannins on the migration of three sheep gastrointestinal nematodes in vitro. Proceedings of the New Zealand Society of Animal Production, 60: 21-25.

34. Molan, A.L., Meagher, L.P., Spencer, P.A., and Sivakumaran, S., (2003). Effect of flavan-3-ols on in vitro egg hatching, larval development and viability of infective larvae of Trichostrongylus colubriformis. Int. J. Parasitol., 33: 1691 - 1698.

35. Molan, A.L., Waghorn, G.C., and Mc Nabb, W.C. (2002). Effect of tannins on egg hatching and larval development of Trichostrongylus colubriformis in vitro. Vet. Rec., 150: 65 - 69.

36. Nfi A., Ndi C., Bayemi R., Tchoumboue J., Njakoi H., Mopoi N., Njakou M., and Sali-Django (1999). The anthelmintic efficacy of 
some indigenous plants in the Northwest province of Cameroon. Rev. Elev. Vét. Pays Trop., 52: 103 - 106.

37. Niezen, J.H., Robertson H.A., Waghorn G.C., and Charleston W.A.G. (1998). Production, faecal egg counts and worm burdens of ewe lambs which grazed six forages. Vet. Parasitol., 80: 15 - 27.

38. Niezen, J.H., Waghorn, G.C. and Charleston, W.A.G. (1998). Establishment and fecundity of Ostertagia circumcincta and Trichostrongylus colubriformis in lambs fed lotus (Lotus pedunculatus) or perennial ryegrass (Lolium perenne). Vet. Parasitol., 78: 13 - 21.

39. Olounladé, A.P. (2005). Effets anthelminthiques des feuilles de Newbouldia leavis testées in vivo sur les nématodes gastrointestinaux Haemonchus contortus et Trichostrongylus colubriformis) chez les moutons Djallonké. Diplôme d'Etudes Approfondies (DEA) de pharmacologie - physiologie, Université de Lomé (Togo), 65p.

40. Olounladé, A.P. (2011). Analyse phytochimique et propriétés anthelminthiques de Newbouldia laevis (P. Beauv.) Seeman ex Bureau et de Zanthoxylum zanthoxylö̈des (Lam.) Zepernick et Timler sur des Nématodes gastro-intestinaux animaux et humains. Thèse de doctorat de l'Université d'Abomey-Calavi, Bénin, 296p

41. Osuji, P.O., Nsahlai, I.V., and Khalili, H. (1993). Fed evaluation. ILCA Manual 5, 40 p.

42. Paolini, V., Bergeaud, J.P., Griez, C., Prevot, F., Dorchies P.H., and Hoste, H. (2003a). Effects of condensed tannins on goats experimentally infected with Haemonchus contortus. Vet. Res., 34: 1 $-9$.

43. Paolini, V., Dorchies, P.H., and Hoste, H. (2003b). Effects of sainfoin hay on gastrointestinal nematode infections in goats. Vet. Rec., 152: $600-601$.

44. Paolini, V., Dorchies P.H., Athanasiadou S., and Hoste, H. (2002). Effets des tannins condensés et des plantes à tannins sur le parasitisme gastro-intestinal par les nematodes chez la chèvre. Renc. Rech. Ruminants, 9 : 411 - 414.

45. Paolini, V., Fouraste, I., Dorchies, P.H., and Hoste, H. (2003c). In vitro measurements of anthelmintic effects of tanneferous plants on third larval of parasitic nematodes of gastrointestinal tract. The $19^{\text {th }}$ international conference of the world association for the advancement of veterinary parasitology. New Orleans? August 10 - 14: 219.

46. Paolini, V., Frayssines, A., De La Farge, F., Dorchies, P.H., and Hoste, H. (2003d). Effects of tannins on goats experimentally infected with Haemonchus contortus. Vet. Parasitol., 113: 253 - 261. 
47. Pauwel, L. and Nzayilu, N. (1993). Guide des arbres et arbustes de la région de Kinshasa Brazzaville Meise jardin botanique National de Belgique et la CEE. Vol 4: 495p.

48. Raynaud, J.P. (1970). Etude de l'efficacité d'une technique de coproscopie quantitative pour le diagnostic de routine et le contrôle des infestations parasitaires des bovins, ovins, équins et porcins. Ann. Parasitol. Hum. Comp., 45: 321-342.

49. Tamboura, H., Kaboré, H. and Yameogo, S.M. (1998). Ethnomédecine vétérinaire et pharmacopée traditionnelle dans le plateau central du Burkina Faso : cas de la province du Passoré. Biotechnol. Agron. Soc. Environ., 2: 181 - 191

50. Varady, M., and Corba, J.L. (1999). Comparison of six in vitro test in determining benzimidazole and levamisole resistance in Haemonchus contortus and Ostertagia circumcinta of sheep? Vet. Parasitol., 80: 239 - 249. 